\title{
Collectivistic coping strategies for distress among Polynesian Americans
}

\author{
G. E. Kawika Allen \\ Brigham Young University, gekawika_allen@byu.edu
}

Timothy B. Smith

Brigham Young University, tbs@byu.edu

Follow this and additional works at: https://scholarsarchive.byu.edu/facpub

Part of the Counseling Psychology Commons

\section{Original Publication Citation}

Allen, G. E., \& Smith, T. B. (2015). Collectivistic coping strategies for distress among Polynesian Americans. Psychological Services, 12(3), 322-329.

\section{BYU ScholarsArchive Citation}

Allen, G. E. Kawika and Smith, Timothy B., "Collectivistic coping strategies for distress among Polynesian Americans" (2015). Faculty Publications. 2031.

https://scholarsarchive.byu.edu/facpub/2031

This Peer-Reviewed Article is brought to you for free and open access by BYU ScholarsArchive. It has been accepted for inclusion in Faculty Publications by an authorized administrator of BYU ScholarsArchive. For more information, please contact ellen_amatangelo@byu.edu. 
Allen, G. E., \& Smith, T. B. (2015). Collectivistic coping strategies for distress among Polynesian Americans. Psychological Services, 12(3), 322-329.

Collectivistic Coping Strategies for Distress among Polynesian Americans 


\begin{abstract}
Previous research has shown that psychological services designed to assist clients in coping with stressful or traumatic events are more effective when aligned with clients' cultural values, practices, and worldviews. However, limited research is available regarding the preferred coping strategies of Polynesian Americans. In examining collectivistic coping styles and their association with previous distress among 94 Polynesian Americans, we found that participants were highly likely to use family support and religion/spirituality to buffer the initial and residual effects of distress, using private emotional outlets, such as psychotherapy, very infrequently. The use of private emotional outlets was associated with decreased impairment from distress, although family support was much more predictive of decreased impairment and positive psychological well-being. Mental health professionals can align their services with the cultural values of Polynesian Americans by accounting for collectivistic coping styles and family dynamics.
\end{abstract}

Keywords: coping, distress, Polynesian Americans 
Collectivistic Coping Strategies for Distress among Polynesian Americans

Research of specific client populations, particularly historically oppressed cultures and ethnicities, can enhance the effectiveness of mental health services with clients otherwise overlooked by the profession (e.g., Heppner, 2006; Marsella \& Yamada, 2000; Pedersen, Draguns, \& Trimble, 2008). Population-specific research informs practitioners about particular mental health needs, conceptualizations, and resources across different populations (Bernal \& Sáez-Santiago, 2006; Smith, Domenech Rodríguez, \& Bernal, 2011). More research must be conducted and attention given to underrepresented groups in order to clarify presenting concerns and best practices to enable professionals to provide state-of-the-art services for specific groups.

Historically, the literature on multicultural psychology has focused primarily on Latinos/as, Asian Americans, African Americans, and Native American Indians-groups also represented in general psychology research (Case \& Smith, 2000). Polynesian Americans have been conspicuously underrepresented in all aspects of psychological research. A few studies have examined Native Hawaiians and/or Pacific Islanders in general (McCubbin, 2006; McCubbin \& Dang, 2010; McCubbin, Ishikawa \& McCubbin, 2007), but very little empirical investigation has specifically focused on Polynesian Americans, particularly on their psychological well-being in the mainland U.S. (Allen, Garriott, Reyes \& Hsieh, 2013; Allen \& Heppner, 2011).

For decades Polynesian Americans have been lumped under the Asian American/Pacific Islander census category, although they represent a group that is distinct culturally, historically, linguistically, and religiously. Research focused on Polynesian Americans not only provides necessary descriptions of unique cultural characteristics, but also facilitates understanding of specific psychological processes in the Polynesian American cultural context (e.g., McCubbin, 
2006). Such research is essential to informing culture-specific adaptations to psychotherapy (Bernal, Jiménez-Chafey, \& Domenech Rodríguez, 2009; Bernal \& Sáez-Santiago, 2006), which have been shown to improve client outcomes in treatment (Benish, Quintana, \& Wampold, 2011; Griner \& Smith, 2006; Smith et al., 2011; Smith \& Trimble, in press).

\section{Collectivistic Coping}

A value of Polynesian Americans that must be considered in psychotherapy is cultural collectivism. Cultural collectivism, distinguished from the individualism characteristic of Western societies, is an interdependent orientation empahsizing connectedness to others, importance of social contexts, and significance and maintenance of relationships (Marcus \& Kitayama, 1991; Singelis, 1994; Yeh, Inman et al., 2006). An important dimension of collectivistic coping is family support (Heppner et al., 2006; Yeh, Inman et al., 2006), which involves families resolving the stresses of life together to ensure that all members are psychologically stable and healthy. A second dimension of collectivism is holistic worldviews, often explicitly including spirituality. Although insufficient research has investigated coping with distress among Polynesian Americans (McCubbin et al., 2007), these two aspects of collectivism have been commonly identified in research with other non-Western populations. A qualitative research study of Asian American family members who experienced the loss of loved ones in the 9/11 tragedy (Yeh, Inman et al., 2006) found that the participants utilized spiritual collectivistic coping, which included interpreting the tragedy through the lens of religious teachings and increasing their religious or spiritual activity by praying, speaking with religious leaders, and attending religious services. Similarly, a large quantitative study (Heppner et al., 2006) found strong empirical evidence for both family support and religious/spiritual coping among East Asians; in contrast, coping strategies not culturally congruent with a collectivistic 
worldview, such as detachment, were associated with greater levels of distress. Psychotherapists should attend to the coping methods used by their clients from collectivistic cultures.

\section{Cultural Disconnections with Mental Health Services}

Notwithstanding the need to attend to clients' cultural worldviews, psychotherapy typically does not address or facilitate collectivistic coping methods (Hall, 2003; TummalaNarra, 2007), such as extensive family discussions or spiritual methods for healing and finding meaning. Hence there is a disconnect between common forms of therapy, which tend to emphasize individualistic methods of coping (Kirmayer, 2007; Smith \& Draper, 2004), and the collectivistic strategies preferred among many non-Western cultures (Hall, 2003), including Polynesian Americans.

The problematic disconnect between individualistic mental health services and collectivistic cultural preferences becomes easily apparent in cases involving distress or trauma (Tummala-Narra, 2007). Individuals respond to distress or trauma with acquired, familiar coping strategies (Aldwin, 2007). So if a client has acquired collectivistic coping strategies but the therapist advocates individualistic methods, such as cognitive therapy, the client may not successfully implement the cognitive therapy or may feel invalidated and thus remain traumatized.

Moreover, individualistic approaches to treatment "are designed to help with single trauma and ignore the cumulative trauma dynamics as well as the collective identity and culturespecific traumas” (Kira, 2010, p. 137). Racial and ethnic minorities experience cumulative collective distress, and they report relatively high levels of trauma (Perilla, Norris, \& Lavizzo, 2002; Switzer et al., 1999; Weems et al., 2010). For example, ethnic minority youth exposed to Hurricane Katrina had higher than expected PTSD symptom rates that did not significantly 
decline over time (Weems et al., 2010). Similarly, among Florida residents exposed to Hurricane Andrew, Latinos/as had the highest rate of PTSD (38\%), followed by African Americans (23\%), with Whites having by far the lowest rate (15\%) (Perilla, Norris \& Lavizzo, 2002). In another study, 94\% of urban African Americans had experienced trauma, with 42\% reporting having PTSD during the previous year (Switzer et al., 1999). These researchers also found relative dissatisfaction with mental health services among the African American clients with PTSD. Thus, it is possible that cultural differences may not only result in increased risk of distress, but also in decreased satisfaction with the treatment provided.

\section{Research Questions}

Although limited research has examined strategies for coping with distress among racial ethnic minorities, virtually none has addressed these issues among Polynesian Americans. Research specific to that population is clearly needed, particularly accounting for collectivistic coping strategies. Therefore, this study was focused to answer the following questions:

1. Which collectivistic coping strategies do Polynesian Americans find most helpful when coping with a distressing event?

2. To what degree are collectivistic coping strategies associated with ameliorating life interference from a distressing event?

3. Which specific collectivistic coping strategies are most strongly associated with experiences of family life among Polynesian Americans?

4. Which specific collectivistic coping strategies are most strongly associated with psychological well-being among Polynesian Americans?

\section{Method}

\section{Participants and Procedure}


As part of a larger study (Allen \& Heppner, 2011), 110 participants were recruited from a suburban city with a high concentration of Polynesian Americans. Participants were asked to complete a survey containing several measures of psychological well-being; data for the present study consisted of the measures of collectivistic coping strategies, distressing event severity, and family home environment that had not been analyzed or reported previously. Ten surveys were excluded due to excessive missing values, and four surveys were removed because the respondents indicated an ethnicity other than Polynesian American. Two surveys were removed because they had extreme scores (three standard deviations above or below the mean), which could compromise the validity of the study (Cohen, Cohen, West, \& Aiken, 2003; Tabachnick \& Fidell, 2007). The final sample consisted of 94 adults (55 women, 37 men, and 2 who did not report gender); the mean age was 31.5, with a range of 18 to 75 years. Of these participants $79 \%$ were of Samoan heritage $(n=75), 17 \%$ reported being multiethnic Polynesian $(n=16), 3 \%$ reported being Native Hawaiian $(n=3)$, and 1\% was of Tongan descent $(n=1)$.

We had previously conducted a statistical power analysis for multiple regression in using procedures outlined by Cohen (1988). Assuming an effect size of $d=.33$, the minimum number of participants required for this study was 84 to achieve statistical power of .80. The obtained sample exceeded that requirement.

\section{Instruments}

Collectivistic Coping Styles (CCS; Heppner et al., 2006). This instrument was developed and validated in three studies among Taiwanese college students. This instrument was chosen due to the prevalence of collectivistic coping in both Taiwanese and Polynesian American individuals and communities. Five factors were confirmed through exploratory and confirmatory factor analyses: (a) acceptance, reframing, and striving (ARS), (b) family support 
(FS), (c) religion-spirituality (RS), (d) avoidance and detachment (AD), and (e) private emotional outlets (PEO). The CCS is rated on a 6-point Likert-type scale: 0 (never used this strategy), 1 ( used but of no help at all), 2 (a little help), 3 (a moderate amount of help), 4 ( $a$ great deal of help), 5 (a tremendous amount of help). The reliability coefficient (Chronbach's alpha) for the total CCS and all five subscales for both the normed and current sample were as follows: total CCS (.87; .80 in this study), acceptance, reframing, and striving (.85; .76 in this study), family support (.86; .85 in this study), religion-spirituality (.90; .85 in this study), avoidance and detachment (.77; .79 in this study), and private emotional outlets (.76; .72 in this study).

Because the CCS is a situation-specific coping inventory, the participants were first asked to select a stressful problem from the following list of distressing events that they might have experienced in their lives: a major accident, violence at home, natural disaster (e.g., earthquake), death or illness of a loved one, personal illness, academic pressure, relationship termination with significant others (e.g., divorce, breakup of romantic relationship), social isolation and peer group ostracism, poverty, unemployment or job loss (including self or family), racial or cultural discrimination, and other (with a space for participants to describe the event).

Results showed the most common stressful event reported by participants was death or illness of a loved, selected from the list by 27 (27\%), followed by multiple stressful events reported by 19 (20\%). Of the remaining participants, 18 (19\%) described an event in the selfreport category of "other” rather than selecting a stressful event provided from the list, 9 (11\%) reported the termination of a relationship with a significant other, and 6 (6\%) reported unemployment or job loss. All other stressful events reported were fairly equally distributed across the listed items. Regarding the level of severity of the stressful event, 41 (44\%) indicated 
that the selected event(s) were extremely stressful, while 21 (22\%) reported moderately stressful, 16 (17\%) reported mildly stressful, 6 (6\%) indicated somewhat stressful, and 10 (11\%) reported not stressful at all. Regarding when the stressful event had occurred, the majority of the participants indicated in recent years. The largest number, 20 (22\%), reported that their stressful event had happened less than 3 months ago, 14 (15\%) reported between 7 and 12 months, 18 (19\%) indicated between 1 and 3 years, and 13 (14\%) marked between 4 and 6 years. When participants reported how often the stressful event had happened, 43 (46\%) reported once, while 23 (24\%) indicated twice, and 15 (16\%) indicated more than five times.

Traumatic Interference Index (TII). The participants were also asked how much the stressor affected four domains of their lives (mood, school/work, interpersonal relationships, and thinking and judgment) at the time it first happened. The following scale was used to rate the degree of interference: 1 (no interference at all), 2 (a little interference), 3 (a moderate amount of interference), 4 (a lot of interference), 5 (a major interference). The Chronbach’s alpha coefficient for this scale in this study was .85 .

Positive Attitudes Toward Living at Home (PALH; Glezer, 1984). Because family cohesion is an important aspect of collectivistic coping, we assessed participants’ perceptions of family life using the PALH. This instrument focuses mainly on evaluations of one's family of origin and the environment in which the individual was raised. A Likert-type scale is used:1 (strongly disagree) to 5 (strongly agree). Six items evaluate family climate: pleasantness living at home (PLH; whether participants felt like the home environment had been pleasant during their youth) and openness to discussing issues at home (ODH; whether participants had felt comfortable discussing problems or important matters openly with family members during their youth). Several items evaluating negative aspects of environment (discomfort, arguments, etc.) 
were reverse scored, such that overall high scores represented positive views of family life. The alpha coefficient reported by the developer of the PALH was .83, and for this study it was .70.

Psychological Well-being scale, Self-Acceptance and Purpose in Life subscales. Ryff and Keyes (1995) developed and validated the Psychological Well-being Scale (PWS) to assess the nature and meaning of people's well-being. Following the exploratory and confirmatory analyses and an analysis of fit, the developers found six main dimensions of psychological wellbeing, which became the subscales of the instrument: Self-Acceptance, Environmental Mastery, Positive Relations, Purpose in Life, Personal Growth, and Autonomy. All were reported to have had evidence of reliability and validity.

In the present study two PWS subscales were used to measure and assess psychological well-being among Polynesian Americans: Self-Acceptance (SA) and Purpose in Life (PL). High scorers on the SA dimension are described as persons who have a positive attitude toward self; acknowledge and accept multiple aspects of self, including positive and negative qualities; and feel positive about past life. Low scorers are described as persons who feel dissatisfied with self, are disappointed with what has occurred in past life, are troubled about certain personal qualities, and wish to be different than they are. The SA is a 14-item subscale with an internal consistency coefficient of .91 in this study. It is rated on a six-point Likert-type scale: 1 (strongly disagree) to 6 (strongly agree). Sample SA items include "I like most aspects of my personality" and "For the most part, I am proud of who I am and the life I lead.”

The Purpose in Life (PL) subscale evaluates individuals’ sense of directedness and meaning in life. Low scores reflect a lack of meaning in life, limited future goals, and absence of direction. The PL has 14 items, with an internal consistency coefficient of .88 in this study. It is rated on the same six-point Likert-type scale as the SA. Some of the PL items are "I have a sense 
of direction and purpose in life" and "Some people wander aimlessly through life, but I am not one of them.”

\section{Results}

Preliminary analyses using descriptive statistics confirmed the normality of the data distributions and range of scores (see Table 1).

\section{Prevalence of Helpful Coping Strategies}

In addressing our research questions, we first sought to identify which of the coping methods had been most helpful for the participants when experiencing distressing events. Means on two of the CCS subscales, Family Support (FS; $M=3.6$ ) and Religion-Spirituality (RS; $M=$ 4.0) were statistically much higher than those reported in the initial development of the CCS (Heppner et al., 2006): FS, $M=2.4, p<.001$; RS, $M=2.2, p<.001$. Thus participants in this sample were much more likely to use and benefit from those two collectivistic coping strategies than the participants involved in the original CCS research. Strong family support and religious and spiritual coping were highly characteristic of this sample of Polynesian Americans.

\section{Bivariate Associations of Coping Strategies with Distress}

We next evaluated the relationship between collectivistic coping and the level of distress experienced at the time of the stressful event, as measured by the TII. Table 2 summarizes the bivariate correlations among study variables. Distress at the time of the event was apparently buffered to some degree by family support and also by avoidance and detachment. Specifically, family support was inversely correlated with all four of the TII subscales, work $(r=-.21, p<$ $.01)$, interpersonal relationships $(r=-.21, p<.01)$, thinking and judgment $(r=-.33, p<.001)$, and mood $(r=-.28, .001)$. Similarly, avoidance and detachment were inversely correlated with three of the TII subscales: school and work $(r=-.24, p<.01)$, thinking and judgment $(r=-.27, p$ 
$<.001)$, and mood $(r=-.24, p<.01)$. The other methods of coping were not consistently associated with impairment experienced at the time of the distress.

\section{Multivariate Associations of Coping Strategies with Distress}

Notwithstanding the straightforward pattern of bivariate correlations, the association between sets of variables is better explained by multivariate analyses, so we examined the overall association between the constructs of collectivistic coping (CCS subscales) and impairment due to distressing events (TII subscales) through canonical correlation (Brown, Hendrix, Hedges, \& Smith, 2011), the appropriate method when comparing sets of variables. This analysis yielded four canonical variates for the two sets of measures because the smaller set consisted of four measures (the four TII subscales). The overall multivariate test for dimensionality reached statistical significance (Wilk’s lambda $=.68, p<.001$ ), with the first canonical function being the only one to reach statistical significance $(p<.05)$. This step of the analysis could be considered similar to the extraction of factors in an exploratory factor analysis (Brown et al., 2011), with only one factor predominating. In other words, the relationship between the collectivistic coping measures and the measures of distress could be explained parsimoniously. The canonical correlation between collectivistic coping (CCS) and distress (TII) was .45; the two constructs shared $20 \%$ of their variance.

The associated canonical loadings are presented in Table 3. These data can be interpreted similar to factor loadings; they represent the degree to which each variable contributes to the canonical function, with values above .30 indicating a meaningful contribution. All four subscales of the TII loaded on the first canonical function. Specifically, distress in thinking and judgment loaded the most highly (-.91), followed by mood (-.82), school and work (-.67), interpersonal relationships (-.44). Thus the first canonical function was a latent variable that 
represented general distress across all four aspects measured, although cognitive and emotional distress (the first two variables) were more prominent than distress in life circumstances (the second two variables), meaning that the reported distress (TII) that was relevant to collectivistic coping (CSS) was more of an internal/personal nature than external/situational, as would be expected of psychological reactions to stressful events.

As can be seen in Table 3, the two subscales of the CCS with the highest canonical loadings were family support (.75) and avoidance and detachment (.73). Those two measures contributed most to the latent variable representing collectivistic coping; however, personal emotional outlets also loaded strongly (.58), with avoidance, reframing, and striving contributing to some degree (.38), but with religion/spirituality contributing less (.23). Thus the multivariate analysis indicated that several aspects of collectivistic coping helped to explain the association with impairment from distress.

\section{Collectivistic Coping Strategies and Family Life}

We next examined the degree to which the collectivistic coping strategies were associated with the family life of the Polynesian American participants. As would be expected, individuals who benefitted from family support when coping with distress also reported discussing matters with members of their family of origin $(r=.33, p<.01)$ and having pleasant experiences while living at home $(r=.31, p<.01)$. However, it was noteworthy that coping using acceptance, reframing, and striving and also using religion/spirituality were similarly positively associated with discussing matters with family members and having pleasant experiences while living at home, with correlations ranging from .21 to .33 (see Table 2).

These findings suggested that the type of coping strategies used and perceived to be helpful do vary as a function of family dynamics. In fact, they were associated with family 
dynamics to nearly the same degree that using family support was associated with family dynamics. Nevertheless, when simultaneously entering all five types of collectivistic coping into a regression model that explained 16\% $(p<.01)$ of the variance in family dynamics (combined PLH and ODH, which were correlated at .49), the only one that remained statistically significant at the univariate level was family support $(b=.26 ; p<.05)$. Religious/spiritual coping and acceptance, reframing, and striving were associated with family dynamics, but due to a high degree of multicollinearity they were partialed out of the relationship after accounting for family support.

\section{Collectivistic Coping and Psychological Well-being}

The collectivistic coping strategies used by this sample of Polynesian Americans were significantly associated with their psychological well-being, as measured by self-acceptance (SA) and purpose in life (PL).

1. Acceptance, reframing, and striving were positively correlated with both SA and PL ( $r$ $=.25$ and .26 , respectively; see Table 2 ).

2. Family support and religion/spirituality were positively associated with PL $(r=.24)$.

3. Both avoidance/detachment and private emotional outlets were negatively associated with both SA and PL (correlations ranged from -.20 to -.33; see Table 2).

Participants' coping styles were clearly associated with their core personal beliefs and outlook on life. However, the key distinction is that acceptance, reframing, and striving, along with family support and religion/spirituality, were more likely to be used by individuals with stronger self-acceptance and purpose in life, whereas avoidance/disengagement and private emotional outlets were more likely to be used by individuals with less self-acceptance and purpose in life. Entering all five types of collectivistic coping in a regression model explained 
34\% ( $p$.001) of the variance in psychological well-being (combined PL and SA, which were correlated at .70). The two coping methods that remained statistically significant at the univariate level were acceptance, reframing, and striving $(b=.38 ; p=.001)$ and private emotional outlets $(b=-.43 ; p<.001)$. The addition of family dynamics (PLH and ODH) to the regression model did not increase the amount of explained variance, which remained at 34\%. Thus collectivistic coping strategies accounted for positive well-being independent of family dynamics.

\section{Discussion}

Psychologists work with individuals in distress. Those individuals vary in their coping methods and in their level of impairment from the distress; both types of variation may be due to contextual factors such as cultural background. Different cultures emphasize different methods for coping (e.g., Heppner et al., 2006). Psychologists should therefore be informed about culture-relevant coping methods. The results of this study provide important information about coping strategies among Polynesian Americans that can and should influence adaptation of treatments to better align with client cultural worldviews (Benish et al., 2011; Smith et al., 2011; Smith \& Trimble, in press).

\section{Family and Religious Support vs. Avoidance/Detachment and Private Outlets}

Specifically, the data indicated that Polynesian American participants most frequently reported that when confronted with stressful situations they benefit from religious and spiritual coping (e.g., praying, meditating, fasting, actively participating in religious services, reading religious texts) and from the support of their family members (e.g., openly discussing problems with them). In fact, the mean perceived helpfulness ratings of religious/spiritual coping and 
family support coping were much higher $(p<.001)$ than the corresponding means reported in the initial development of the CCS (Heppner at al. 2006).

The participants in this study reported that using private emotional outlets was the least helpful coping method. Perhaps, participants were less likely to seek opportunities to share their problems in private with others. When considered together, these findings could suggest that Polynesian Americans are also unlikely to spontaneously seek professional mental health services (Allen et al., 2013; Allen \& Heppner, 2011) due to perhaps stigma and cultural difference in seeking help, but would be much more likely to address their problems within their family and their religious/spiritual community. Therapists who aim to enhance the cultural compatibility of the services they provide should seek ways to reduce reported barriers to receiving professional treatment (Smith \& Trimble, in press). And therapists working with Polynesian American clients can appropriately inquire about communal supports and integrate those into treatment (Bernal \& Sáez-Santiago, 2006). Such cultural adaptations should improve client retention and outcome (Bernal et al., 2009; Smith \& Trimble, in press).

An important finding is that collectivistic coping strategies were moderately associated with decreased levels of reported impairment from distress. In particular, family support and avoidance and detachment explained much of that association, with private emotional outlets also contributing. This finding qualifies the previously reported finding of self-reported benefits from using each coping strategy. Even though the Polynesian American clients reported (a) somewhat infrequent benefits from avoidance and detachment and (b) very infrequent benefits from private emotional outlets, these coping strategies were associated with decreased impairment from distress. 
Avoidance and detachment may have a negative stigma in contemporary society, making Polynesian Americans reluctant to rate this strategy as helpful even though it appears to have actually helped them. Alternatively, avoidance and detachment may have been strategies used in more serious distress, so that the apparent benefits were exaggerated in the correlational data since individuals with mild to moderate distress could be less likely to use avoidance and detachment. Future research is needed to sort out the plausible causal pathways. The same could be said about the use of private emotional outlets like psychotherapy. Those outlets appeared to be associated with decreased impairment from distress, even though participants infrequently rated them as being helpful. It is possible that the individuals who availed themselves of private emotional outlets were indeed benefitted, but because the majority did not use them, the overall rating for the strategy was low.

Overall, family support was perceived to be a very helpful coping strategy; it was associated with lower levels of impairment from distress and with a greater sense of purpose in life. As would be expected, Polynesian Americans who reported pleasant experiences living at home (e.g., fewer arguments, more relationship-building moments) were the most likely to report benefitting from family support during times of distress. They were also more likely to benefit from spiritual and religious coping methods. In contrast, individuals who reported less positive home environments were more likely to report using and benefiting from avoidance, detachment, and a tendency to be private about emotions. Thus the degree of family cohesion is associated with type of coping preferred. Therapists should therefore evaluate the degree of family cohesion before assuming that a particular Polynesian American client has strong family bonds just because Polynesian Americans typically do. Furthermore, the coping methods of family support and religious/spiritual strategies also increased the likelihood of greater well-being, 
particularly for those experiencing self-acceptance and purpose in life. In particular, these findings underscore the association between Polynesian Americans' coping strategies and family life. In that regard, these results are similar to those found with other racial and ethnic minority groups in the U.S. (Yeh, Inman et al., 2006; Yeh, Arora et al., 2006). Additional research is needed with other Polynesian American samples to confirm these findings.

\section{Limitations}

Several limitations of this study deserve consideration. First of all, the cross-sectional research design that was used cannot establish causality, so interpretations of the correlational data remain tentative. Future research should seek to confirm the causal direction of the associations observed. Second, study participants were predominantly of Samoan ancestry and did not represent Polynesian Americans as a whole. Additional research is needed with other Polynesian groups, including Native Hawaiians, Tongans, Fijians, and Maoris. Third, several of the older participants in this study spoke English as a second language, so a few of the participants asked a younger Samoan to explain some of the English words on the survey. Future research would benefit from preparing copies of the inventories in the native languages of the participants. Fourth, even though 94 participants were adequate for data analysis, a larger sample would have helped to make the findings more robust. Nevertheless, to our knowledge this is one of very few studies in the psychology literature to involve more than 90 Polynesian Americans.

\section{Implications for Practice}

The results of this study highlight the necessity for therapists working with Polynesian Americans to explicitly attend to collectivistic coping strategies (particularly family support and religion/spirituality) and to account for probable associations with family dynamics, 
psychological well-being, and distress. If therapists are not appropriately sensitive to clients' cultural contexts when they engage in psychotherapy with Polynesian Americans who hold collectivistic worldviews and who act in close coordination with their families and religious communities, those therapists may decrease the likelihood that Polynesian clients will participate in professional mental health services (Cervantes \& Parham, 2005; Smith \& Trimble, in press). Results are more beneficial if therapists consider clients’ preferred methods for handling distress and maintaining psychological well-being as they attend to cultural considerations (Bernal et al., 2009).

Although mental health practices are being effectively adapted to better meet the needs of many ethnic minority groups, the benefits of multicultural and cross-cultural research need to be extended to Native American Indians, Alaska Natives, Polynesian Americans, and other groups unrepresented in the literature (e.g., Duran \& Duran, 1995; Richards \& Bergin, 2014). This research on Polynesian Americans’ experiences with collectivistic coping, distress, and psychological well-being was conducted to facilitate appropriate cultural adaptations to mental health services for this underserved population (Benish et al., 2011; Smith et al., 2011; Smith \& Trimble, in press).

Currently the field of psychology understands very little about the experiences of Polynesian Americans. In recent years a few studies have investigated the well-being, identity, trauma, and resilience of Native Hawaiians (McCubbin, 2006; McCubbin \& Dang, 2010; McCubbin, Ishikawa \& McCubbin, 2007). However, additional research on other Polynesian American groups (e.g., Tongan, Samoan, Fijian, Maori) living in the U.S. is strongly encouraged to add to our knowledge of the cultural contexts, identities, acculturation, and psychological well-being of these cultural groups. 


\section{References}

Aldwin, C. M. (2007). Stress, coping, and development: An integrative perspective. New York: Guilford.

Allen, G. E. K., Garriott, P. O., Reyes, C. J., \& Hsieh, C. (2013). Racial identity, phenotype, and self-esteem among biracial Polynesian/White individuals. Family Relations, 62, 8291.

Allen, G. E. K., \& Heppner, P. P. (2011). Religiosity, coping, and psychological well-being among Latter-Day Saint Polynesians in the U.S. Asian American Journal of Psychology, 2(1), 13.

Benish, S. G., Quintana, S., \& Wampold, B. E. (2011). Culturally adapted psychotherapy and the legitimacy of myth: A direct-comparison meta-analysis. Journal of Counseling Psychology, 58(3), 279-289.

Bernal, G., Jiménez-Chafey, M. I., \& Domenech Rodríguez, M. M. (2009). Cultural adaptation of treatments: A resource for considering culture in evidence-based practice. Professional Psychology: Research and Practice, 40(4), 361- 368.

Bernal, G., \& Sáez-Santiago, E. (2006). Culturally centered psychosocial interventions. Journal of Community Psychology, 34(2), 121-132.

Brown, B. L., Hendrix, S. B., Hedges, D. W., \& Smith, T. B. (2011). Multivariate analysis for the biobehavioral and social sciences: A graphical approach. Hoboken, NJ: WileyBlackwell.

Case, L., \& Smith, T. B. (2000). Ethnic representation in a sample of the literature of applied psychology. Journal of Consulting and Clinical Psychology, 68(6), 1107-1110.

Cervantes, J.M., \& Parham, T. A. (2005). Toward a meaningful spirituality for people of 
color: Lessons for the counseling practitioner. Cultural Diversity and Ethnic Minority Psychology, 11(1), 69-81.

Cohen, J. (1988). Statistical power analysis for the behavioral sciences (2nd ed.). Manwah, NJ: Lawrence Erlbaum Associates.

Cohen, J., Cohen, P., West, S. G., \& Aiken, L.S., (2003). Applied multiple regression/correlation analysis for the behavioral sciences ( $3^{\text {rd }}$ ed.). Manwah, NJ: Lawrence Erlbaum Associates.

Duran, E., \& Duran, B. (1995). Native American postcolonial psychology. Albany, NY: SUNY Press.

Glezer, H. (1984). Antecedents and correlates of marriage and family attitudes in young Australian men and women. In Social change and family policies: Key papers of the XXth International CFR Seminar. Melbourne, AU: Australian Institute of Family Studies.

Hall, G. C. N. (2003). The self in context: Implications for psychopathology and psychotherapy. Journal of Psychotherapy Integration, 13(1), 66-82.

Heppner, P. P. (2008). Expanding the conceptualization and measurement of applied problem solving and coping: From stages to dimensions to the almost forgotten cultural context. American Psychologist, 63(8), 805-816.

Heppner, P., Heppner, M. J., Lee, D.-g., Wang, Y.-W., Park, H.-j., \& Wang, L.-f. (2006). Development and validation of a collectivist coping styles inventory. Journal of Counseling Psychology, 53(1), 107-125.

Khalili, S., Murken, S., Reich, S. H., Shah, A. A., \& Vahabzadeh, A. (2002). Religion 
and mental health in cultural perspective: Observations and reflections after the first international congress on religion and mental health. International Journal for the Psychology of Religion, 12, 217-237.

Kira, I. A. (2010). Etiology and treatment of post-cumulative traumatic stress disorders in different cultures. Traumatology, 16(4), 128-141.

Kirmayer, L. J. (2007). Psychotherapy and the cultural concept of the person. Transcultural Psychiatry, 44(2), 232-257.

McCubbin, L. D. (2006). The role of indigenous family ethnic schema on well-being among Native Hawaiian families. Contemporary Nursing Journal: Community \& Family Health, 23, 170-180.

McCubbin, L. D., Ishikawa, M., \& McCubbin, H. I. (2007). Kanaka maoli: Native Hawaiians and their testimony of trauma and resilience. In A. Marsella, J. Johnson, P. Watson, \& J. Gryczynski (Eds.), Ethnocultural perspectives on disaster and trauma: Foundations, issues and applications (pp. 271-298). New York: Springer.

McCubbin, L.D., \& Dang, T. (2010). Native Hawaiian identity and measurement: An ecological perspective on indigenous identity development. In J. G. Ponterotto, J. M. Casas, L. A. Suzuki, \& C. M. Alexander (Eds.), Handbook of multicultural counseling ( $3^{\text {rd }}$ ed.). Thousand Oaks, CA: Sage.

Pedersen, P. B., Draguns, J. G., Lonner, J .L., \& Trimble, J. E. (2008). Counseling across cultures ( $6^{\text {th }}$ ed.). Thousand Oaks, CA: Sage.

Perilla, J. L., Norris, F. H., \& Lavizzo, E. A. (2002). Ethnicity, culture, and disaster response: Identifying and explaining ethnic differences in PTSD six months after Hurricane Andrew. Journal of Social and Clinical Psychology, 21(1), 20-45. 
Richards, P. S., \& Bergin, A. E. (2014). Handbook of psychotherapy and religious diversity ( $2^{\text {nd }}$ ed.). Washington, DC: American Psychological Association.

Ryff, C. D., \& Keyes, C. L. M. (1995). The structure of psychological well-being revisited. Journal of Personality and Social Psychology, 69, 4, 719-727.

Smith, T. B., Domenech Rodríguez, M., \& Bernal, G. (2010). Culture. Journal of Clinical Psychology: In Session, 67, 1-10.

Smith, T. B., \& Trimble, J. E. (in press). Multicultural psychology foundations: A synthesis of scholarship on race and ethnicity. Washington, DC: American Psychological Association.

Sue, D. W., \& Sue, D. (2012). Counseling the culturally diverse: Theory and practice (6 ${ }^{\text {th }}$ ed.). Hoboken, NJ: Wiley.

Switzer, G. E., Dew, M. A., Thompson, K., Goycoolea, J. M., Derricott, T., \& Mullins, S. D. (1999). Posttraumatic stress disorder and service utilization among urban mental health center clients. Journal of Traumatic Stress, 12(1), 25-39.

Tabachnick, B. G., \& Fidell, L.S. (2007). Using multivariate statistics (5 ${ }^{\text {th }}$ ed.). New York: Pearson.

Tummala-Narra, P. (2007). Conceptualizing trauma and resilience across diverse contexts: A multicultural perspective. Journal of Aggression, Maltreatment \& Trauma, 14(1), 33-53.

Weems, C. F., Taylor, L. K., Cannon, M. F., Marino, R. C., Romano, D. M., Scott, B. G., \& Triplett, V. (2010). Post traumatic stress, context, and the lingering effects of the Hurricane Katrina disaster among ethnic minority youth. Journal of Abnormal Child Psychology, 38(1), 49-56.

Yeh, C. J., Arora, A. K., \& Wu, K. A. (2006). A new theoretical model of collectivistic 
coping. In P. T. P Wong \& L. C. J. Wong (Eds.), Handbook of multicultural perspectives on stress and coping (pp. 56-60). New York: Springer.

Yeh, C. J., Inman, A. C., Kim, A. B., \& Okubo, Y. (2006). Asian American families’ collectivistic coping strategies in response to 9/11. Cultural Diversity and Ethnic Minority Psychology, 12(1), 134-148.

Table 1

Means and Standard Deviations of the CCS and PALH.

$\begin{array}{llll}\text { Measure } & M & S D & \text { Skewness }\end{array}$


CCS

$\begin{array}{ccccc}\text { ARS } & 3.30 & .80 & -.30 & -.07 \\ \text { FS } & 3.60^{*} & 1.11 & -.97 & .85 \\ \text { RS } & 4.04^{*} & 1.16 & -1.60 & 2.23 \\ \text { AD } & 2.32 & 1.21 & -.06 & -.58 \\ \text { PEO } & 1.70 & 1.31 & .50 & -.50 \\ \text { PALH } & 3.1 & .39 & 1.60 & 5.90\end{array}$

Note. CCS = Collectivistic Coping Scale; ARS = Acceptance, Reframing, and Striving; FS = Family Support; RS = Religion/Spirituality; AD = Avoidance and Detachment; PEO = Private Emotional Outlets. For the CCS, the $M$ and $S D$ indicate the helpfulness ratings for the specific coping strategy which was calculated from only those who used the strategy. PALH = Positive Attitudes about Living at Home. The significant mean difference compared to normative samples was $* p<.001$. 
Table 2

Bivariate Correlations of Study Variables

\begin{tabular}{|c|c|c|c|c|c|c|c|c|c|c|c|c|c|}
\hline Variable & 1 & 2 & 3 & 4 & 5 & 6 & 7 & 8 & 9 & 10 & 11 & 12 & 13 \\
\hline 1. ARS & 1 & & & & & & & & & & & & \\
\hline 2. FS & $.46 * *$ & 1 & & & & & & & & & & & \\
\hline 3. RS & $.28 * *$ & $.53^{* *}$ & 1 & & & & & & & & & & \\
\hline 4. $\mathrm{AD}$ & $.36 * *$ & .15 & -.08 & 1 & & & & & & & & & \\
\hline 5. $\mathrm{PEO}$ & $.29 * *$ & $.31^{* *}$ & .06 & $.44^{* *}$ & 1 & & & & & & & & \\
\hline 6. TII-Then: M & -.07 & $-.28 * *$ & -.13 & $-.24 *$ & $-.21 *$ & 1 & & & & & & & \\
\hline 7. TII-Then: S/W & -.18 & $-.21 *$ & .08 & $-.24 *$ & -.17 & $.49 * *$ & 1 & & & & & & \\
\hline 8. TII-Then: TJ & -.11 & $-.33 * *$ & -.14 & $-.27 * *$ & -.20 & $.64 * *$ & $.44 * *$ & 1 & & & & & \\
\hline 9. TII-Then: IR & .06 & $-.21 *$ & -.04 & -.04 & -.08 & $.48 * *$ & $.51 * *$ & $.49 * *$ & 1 & & & & \\
\hline 10. SA & $.25 *$ & .16 & .13 & $-.21 *$ & $-.30 * *$ & .07 & .15 & -.13 & .13 & 1 & & & \\
\hline 11. PL & $.26^{*}$ & $.24^{*}$ & $.24^{*}$ & $-.20 *$ & $-.33 * *$ & .07 & .16 & -.05 & .12 & $.70 * *$ & 1 & & \\
\hline 12. PLH & .16 & $.31^{* *}$ & $.27^{* *}$ & .08 & .12 & -.18 & -.18 & -.08 & -.04 & -.04 & -.07 & 1 & \\
\hline 13. $\mathrm{ODH}$ & $.30 * *$ & $.33^{* *}$ & $.21^{*}$ & .07 & .07 & -.14 & -.09 & -.16 & -.09 & .10 & .08 & $.49 * *$ & 1 \\
\hline
\end{tabular}

Note. Correlations above represent participants' reported scores on ARS = Acceptance, Reframing, and Striving; FS = Family Support; RS = Religion/Spirituality; $\mathrm{AD}=$ Avoidance and Detachment; PEO = Private Emotional Outlets; TII Then = interference in general at the time the event happened; TII Now = interference in general at the present time $(\mathrm{M}=$ Mood, $\mathrm{S} / \mathrm{W}=$ School and Work, TJ $=$ Thinking and Judgment, $\mathrm{R}=$ Interpersonal Relationships); SA = Self-Acceptance; PL = Purpose in Life; PLH = Pleasant Living at Home; ODH; Openness to Discussing Important Matters at Home. ${ }^{*} p<.05 .{ }^{* *} p<.01$. 
Table 3

Canonical Correlation Data for Measures of Collectivistic Coping and Impairment from Distress.

Variable

Canonical Loading

Impairment from Distress

Mood

School \& Work

$-.67$

Thinking \& Judgment

Interpersonal Relationships

$-.44$

\section{Collectivistic Coping}

Acceptance, Reframing, \& Striving .38

Family Support .75

Religion/Spirituality

Avoidance \& Detachment .73

Private Emotional Outlets .54 